\title{
SOME NOTIONS AND PROBLEMS OF GAME THEORY
}

\author{
J. C. C. MCKINSEY
}

The theory of games is a relatively new branch of mathematics, which treats of situations involving conflict among rational agents. In a typical problem to which the theory is applicable, each of several people can to some extent, but only partially, influence the outcome of a certain event; no one of them by himself can determine the outcome completely; and they differ in their preferences among the various possible outcomes: the theory of games is then concerned with the problem of what each person should do in order to maximize his expectation of good.

The theory was originally developed-first by von Neumann, ${ }^{1}$ and later by Morgenstern and von Neumann ${ }^{2}$ - to provide a mathematical basis for economics. More recently it has been successfully applied to problems of military tactics. ${ }^{3}$ The mathematical statisticians, ${ }^{4}$ finally, have found that some of the fundamental notions of this theory are extremely useful for their discipline: procedures analogous to those used in the theory of games, if they do not yet tell the statistician exactly what he ought to do, have at least taught him that certain things are better left undone.

In this lecture I shall summarize the mathematical aspects of this theory. The literature of the subject is already too extensive for me to be able to cover it completely in the time at my disposal, but I hope to explain some of the important notions involved, and to indicate some of the outstanding problems.

Before going to a general description of games it is well to explain some technical terminology. In the theory of games one means by a game, roughly speaking, a body of rules which specify unambiguously what, under various conditions, each of certain persons, called the players of the game, is permitted to do, what chance devices are

An address delivered before the Pasadena meeting of the Society, December 1, 1951, by invitation of the Committee to Select Hour Speakers for Far Western Sectional Meetings; received by the editors November 29, 1951.

${ }^{1}$ See von Neumann [19]. (The numbers in brackets refer to items in the bibliography.)

2 See von Neumann and Morgenstern [20].

${ }^{3}$ For a nontechnical account of the relation of the theory of games to military matters, see McDonald [15] and [16]. For a detailed analysis of an interesting problem in this domain (the problem of how an airplane should cruise in searching for a submarine) see Morse [17].

${ }^{4}$ For the application of the theory of games to statistical problems, see Arrow, Blackwell, and Girshick [1], and Wald [25] and [26]. 
to be used, and the like. A play of a game, on the other hand, is a particular way of playing the game from beginning to end. Thus a chess tournament consists of a number of plays of the game of chess.

What are called finite games can conveniently be represented with the help of topological trees. ${ }^{5}$ I shall give an example of such a representation, for the case of a certain three-person game. Suppose that the first move of the game is made by player $P_{1}$, who has to choose from among four alternatives. We represent this situation by the bottom part of Figure I, where the "1" at the lowest point of the figure indicates that the first move is made by $P_{1}$, and the four rising lines indicate that $P_{1}$ has four alternatives at this move.

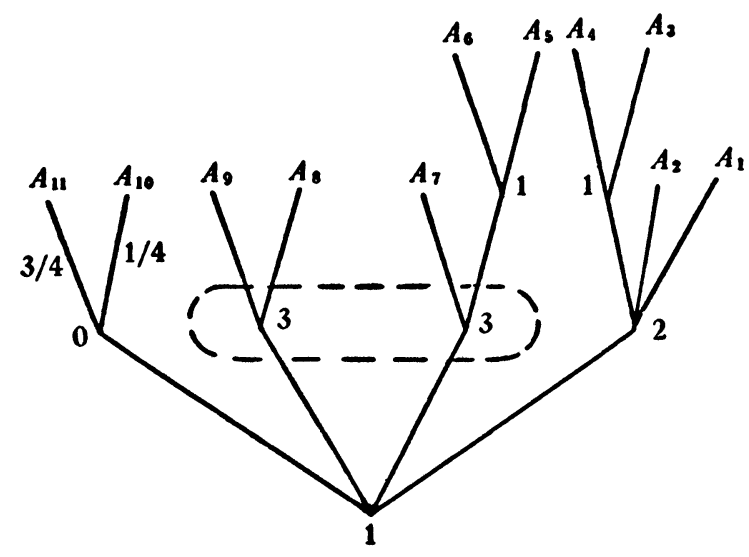

FIG. I

We number the alternatives at this first move (and, indeed, at each of the moves) in a counterclockwise sense with roman numerals. Thus alternative I at the first move is the one corresponding to the segment leading to the vertex marked " 2 " in the figure.

Now suppose that, if $P_{1}$ chooses I on this first move, then the next move is to be made by player $P_{2}$, who in turn has a choice from among three alternatives; and suppose that in addition it is given that if $P_{1}$ chooses II or III on the first move, then the next move is made by player $P_{3}$, who in either case is to choose from two alternatives. This is indicated in Figure I by putting a " 2 " at the end of the segment corresponding to the first choice by $P_{1}$, drawing three lines upward from this point, by putting a " 3 " on the vertices corresponding to

b An exact definition of a game was first given in von Neumann and Morgenstern [20, Chapter II]; but Kuhn, in [12], gave a much simpler definition. My discussion is based on that of Kuhn. 
moves II and III of $P_{1}$, and drawing two lines upward from each of these points.

Suppose, moreover, that, if $P_{1}$ chooses IV on the first move, then the next move is made by chance-an "umpire," let us say, chooses from two alternatives by means of some chance device-and suppose that the chance device is such that it assigns probability $1 / 4$ to the first chance alternative, and $3 / 4$ to the second. We represent this in Figure I by putting a " 0 " at the vertex corresponding to the move made by chance; and putting " $1 / 4$ " and " $3 / 4$ " on the appropriate lines rising from this vertex.

Finally, let us suppose that if $P_{1}$ chooses I and $P_{2}$ chooses III, or if $P_{1}$ chooses II and $P_{3}$ chooses $I$, then the next move is to be made by $P_{1}$ again; that he then has a choice of two alternatives; and that these are the only possible moves in the game. Then the tree in Figure I represents the complete structure of the moves of the game.

When games are represented in this way, we see that any unicursal path from the bottom to the top of the tree represents a play of the game; clearly there are just as many plays as there are top points of the tree. Thus there are precisely eleven possible ways of playing the game represented in Figure I.

It is apparent that, in order to describe the game, it is also necessary to state what will be the payments to or from the various players in case the game terminates at the various points $A_{1}, \cdots, A_{11}$. This can be done by giving a function $H$, which is defined over $A_{1}, \cdots, A_{11}$, and assumes vectors $\langle x, y, z\rangle$ as values; where, for instance, if

$$
H\left(A_{6}\right)=\langle 5,3,-8\rangle \text {, }
$$

this means that, in case the play terminates at $A_{6}$, then $P_{1}$ and $P_{2}$ will be paid 5 and 3 respectively, and 8 will be taken away from $P_{3}$.

In order to complete the description of the game, however, it is also necessary to specify how much the players know about the previous choices at the time they make their moves. (This specification is clearly very important, since the absence of information is, generally speaking, a handicap in playing a game.) Thus suppose, for instance, that when $P_{\mathbf{3}}$ makes his choice he does not know whether $P_{1}$ has chosen II or III on his first move; we can indicate this, as in Figure I, by enclosing the two corresponding points in a region bounded by a dotted line.

It can even happen that $P_{1}$, on his second move, does not "remember" what he did on his first move. Such a situation can be realized in practice by making $P_{1}$ consist of a "team" of two men, who are kept 
isolated from each other, so that the second man, when it is his time to move, does not know what the first man has done. Such a situation would be represented graphically by enclosing within a dotted line the two uppermost vertices of Figure I.

We call such a set of vertices among which a player cannot distinguish when he makes his move an information set. The totality of information sets constitutes a partition of the moves of the game (i.e., of the vertices of the tree); if a player at a certain point is completely informed about the past course of the play, the corresponding information set contains only the single point-in which case we usually omit the dotted line.

If each information set contains just one vertex, we say that the game is one with perfect information. Thus a game with perfect information is one in which each player, at the time when he makes each of his moves, is completely informed about the past course of the play.

In general, an $n$-person finite game is one that can be represented as above by a finite tree. The partition of the vertices into information sets is subject to the obvious restrictions that all the vertices in a given information set must correspond to the same player, and must present the same number of alternatives. In addition it is convenient to impose the following condition:

Condition A. No information set intersects any play in more than one point.

If Condition A is not satisfied, certain difficulties arise, which will be discussed later.

An enormous simplification of the theory of finite games was effected by von Neumann's introduction of the notion of a strategy. By a strategy for a player is meant a function which is defined over the class of his information sets, and for each information set picks out one of the available alternatives. Thus, for the game represented in Figure I, let $\alpha$ be the set consisting of the bottom vertex, and let $\beta$ be the set consisting of the two top vertices; then a strategy for player $P_{1}$ is a function $f$ such that

$$
f(\alpha) \in\{\text { I, II, III, IV }\}
$$

and

$$
f(\beta) \in\{I, I I\} .
$$

Thus $P_{1}$ has eight strategies for this game. The number of strategies for a finite game is of course always finite.

Now imagine that, before starting a play of a game, each player 
picks a strategy and plays according to it. Then, if there are no chance moves involved in the game, the outcome, including what payments will be made, is already determined; thus the players might as well pick their strategies, and then leave to a computing machine the task of calculating the outcome. On the other hand, if the game involves also chance moves, then a choice of strategies by the players does not completely determine the outcome, but it at least determines the expectation of each player; here again, then, after picking their strategies the players can leave the actual play to a machine-though now we shall have to suppose that the machine is provided with suitable chance mechanisms (or with tables of random numbers) which it will employ at appropriate points in the play.

In terms of this notion of strategies, we can now describe, corresponding to a given finite game, an equivalent game of much simpler character. For each player $P_{i}(i=1, \cdots, n)$, let the set of strategies available to $P_{i}$ be $\alpha_{i}$. Let $M_{1}, \cdots, M_{n}$ be functions such that, when player $P_{1}$ chooses strategy $x_{1}$, player $P_{2}$ chooses strategy $x_{2}$, and so on, then the amount

$$
M_{i}\left(x_{1}, \cdots, x_{n}\right)
$$

is the expectation of the $i$ th player. Then the given game is equivalent to the following $n$-move game: on the first move $P_{1}$ chooses an element $x_{1}$ from $\alpha_{1}$; on the second move $P_{2}$, without being informed what choice $P_{1}$ has made, chooses an element $x_{2}$ from $\alpha_{2} ; \cdots$; and on the last move, $P_{n}$, without being informed what choices have been made by the other $n-1$ players, chooses an element $x_{n}$ from $\alpha_{n}$. After all the choices have been made, player $P_{i}$ (for $i=1, \cdots, n$ ) is paid the amount

$$
M_{i}\left(x_{1}, \cdots, x_{n}\right) .
$$

This new form of the game is called the normalized form; the form considered earlier (where there can be many moves, chance moves, etc.) is called the extensive form. The functions $M_{1}, \cdots, M_{n}$ are called the payoff functions of the game in normalized form.

A game in normalized form, with payoff functions $M_{1}, \cdots, M_{n}$, is called zero-sum if for every choice $x_{1}, \cdots, x_{n}$ of strategies for the $n$ players we have

$$
\sum_{i=1}^{n} M_{i}\left(x_{1}, \cdots, x_{n}\right)=0 .
$$

Thus a zero-sum game is one where the sum of the payments made, at least on the average, is zero. 
It is clear that the normalized form of a game is zero-sum if the sum of the payments to the various players is zero for each possible outcome. This condition is not necessary, however, if the game in extensive form involves chance moves, since the payoff functions are defined as expectations.

All the familiar parlor games are zero-sum, since wealth is neither created nor destroyed in the course of playing them-but is merely transferred from one player to another. But this is not to say that only zero-sum games are important. For it appears that economic processes, when considered as games, are ordinarily not zero-sum, since wealth is sometimes created during these processes; and in military processes wealth is often destroyed. ${ }^{6}$

Although the theory of non-zero-sum games is extremely important in practical applications, it appears very difficult to formulate useful concepts for this theory; and the situation is similar with respect to games with more than two players. The conceptual part of the theory of zero-sum, two-person games, on the other hand, is already in a very satisfactory state; and this theory presents a great variety of exactly formulated mathematical problems, some of which turn out to be quite difficult. During the last few years the attention of mathematicians interested in game theory has accordingly been largely devoted to problems about zero-sum two-person games; I want to review very briefly some of the known results in this domain.

Let $\Gamma$ be a (finite) zero-sum two-person game in normalized form. We can suppose that the strategies of $P_{1}$ are represented by the numbers

$$
1, \cdots, m \text {, }
$$

and those of $P_{2}$ by

$$
1, \cdots, n \text {. }
$$

Moreover, there are two functions $M$ and $M^{\prime}$ such that, if $P_{1}$ chooses strategy $i$ and $P_{2}$ chooses strategy $j$, then $P_{1}$ receives

$$
M(i, j)
$$

and $P_{2}$ receives

$$
M^{\prime}(i, j) .
$$

${ }^{6}$ It should be remarked that in military situations it of ten becomes exceedingly difficult to state what sort of assumptions should be made about the utility of the objects created or destroyed -and one is puzzled how to compare the utility scales of the contestants. Thus in such situations it is not always easy to say whether we are dealing with a zero-sum game or not. 
Since $\Gamma$ is zero-sum we have, for all $i \leqq m$ and $j \leqq n$,

$$
M(i, j)+M^{\prime}(i, j)=0 \text {, }
$$

and hence

$$
M^{\prime}(i, j)=-M(i, j)
$$

Thus we can say simply that $P_{1}$ receives $M(i, j)$ and $P_{2}$ receives $-M(i, j)$. Hence the game is determined by the "payoff matrix"

$$
\left(\begin{array}{ccc}
M(1,1) & \cdots & M(1, n) \\
\vdots & & \vdots \\
M(\dot{m}, 1) & \cdots & M(\dot{m}, n)
\end{array}\right)
$$

if $P_{1}$ chooses the $i$ th row and $P_{2}$ chooses the $j$ th column, then $P_{2}$ is to pay $P_{1}$ the amount $M(i, j)$.

Now for any element $i$ which $P_{1}$ may choose, he can be sure of getting at least

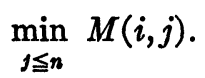

Since $P_{1}$ is at liberty to choose $i$, therefore, he can make his choice in such a way as to insure that he gets at least

$$
\max _{i \leqq m} \min _{j \leqq n} M(i, j) \text {. }
$$

Similarly, $P_{2}$ can make his choice in such a way that $P_{2}$ is sure to get at least

$$
\max _{\mathfrak{n} n} \min _{i \leqq m}-M(i, j),
$$

and hence so that $P_{1}$ will get at most

$$
-\max _{j \leqq n} \min _{i \leqq m}-M(i, j),
$$

which equals

$$
\min _{1 \leqq n} \max _{i \leqq m} M(i, j)
$$

If it happens that

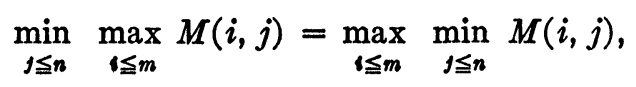

then $P_{1}$ can himself play so as to get this common value (which we denote by " $v$ "), and $P_{2}$ can keep $P_{1}$ from getting more than $v$. In 
this case, there are optimum strategies $i_{0}$ and $j_{0}$ for the two players such that, for all $i$ and $j$,

$$
M\left(i, j_{0}\right) \leqq M\left(i_{0}, j_{0}\right) \leqq M\left(i_{0}, j\right)
$$

and

$$
M\left(i_{0}, j_{0}\right)=\text {. }
$$

Thus $P_{1}$, unless he has good reason to believe that $P_{2}$ will behave in some particular foolish way, cannot do better than to choose $i_{0}$; and similarly, $P_{2}$ cannot do better than to choose $j_{0}$.

It is easily shown that conditions (1) and (2) are equivalent: thus condition (1) is satisfied if and only if the matrix of the game has a saddle-point-i.e., an element which is at the same time the minimum of its row and the maximum of its column. The matrix

$$
\left(\begin{array}{rr}
2 & 3 \\
1 & -5
\end{array}\right)
$$

for example, has a saddle-point in its upper left-hand corner; hence $P_{1}$ can expect to get at least 2 in this game (by choosing the first row), and $P_{2}$ can keep $P_{1}$ from exceeding 2 (by choosing the first column).

It is interesting to note in this connection that Morgenstern and von Neumann have shown (in [20, pp. 112-124]) that the matrix of the normalized form of every two-person, zero-sum game with complete information has a saddle-point. Thus there exist optimum strategies for playing chess, for example, or backgammon. The theorem does not apply to games like bridge or poker, however, where one does not know what cards have been dealt one's opponents.

On the other hand, not every matrix has a saddle-point; a simple example is the following:

$$
\left(\begin{array}{rr}
1 & -1 \\
-1 & 1
\end{array}\right)
$$

The question then arises whether there are nevertheless optimum ways of playing such games. The answer is affirmative, provided we introduce a new notion: namely, that of using a chance mechanism for the choice of strategies.

Suppose that, in the game whose matrix is

$$
\left(\begin{array}{ccc}
M(1,1) & \cdots & M(1, n) \\
\vdots & & \vdots \\
M(m, 1) & \cdots & M(m, n)
\end{array}\right),
$$


the first player, instead of choosing the $m$ strategies available to him in some predetermined manner, selects them at random with probabilities $x_{1}, \cdots, x_{m}$, and that the second player selects his strategies with probabilities $y_{1}, \cdots, y_{n}$. This means that $P_{1}$ chooses a vector $X=\left\langle x_{1}, \cdots, x_{m}\right\rangle$ from the set $\delta_{m}$ of all vectors $\left\langle z_{1}, \cdots, z_{m}\right\rangle$ whose components satisfy

$$
z_{i} \geqq 0 \text { for } i=1, \cdots, m, \sum_{i=1}^{m} z_{i}=1 ;
$$

and similarly that $P_{2}$ chooses a vector $Y=\left\langle y_{1}, \cdots, y_{n}\right\rangle$ from the analogously defined set $S_{n}$. (Elements of $S_{m}$ and $S_{n}$ are called mixed strategies; we sometimes call the original strategies $1, \cdots, m$ and $1, \cdots, n$ pure strategies.) Then the expectation $E(X, Y)$ of the first player is given by the equation

$$
E(X, Y)=\sum_{j=1}^{n} \sum_{i=1}^{m} M(i, j) x_{i} y_{j} .
$$

Reasoning as we did in the case of pure strategies, we see that, by playing properly, $P_{1}$ can insure that his expectation will be at least

$$
\max _{X \in \mathcal{S}_{m}} \min _{Y \in \mathcal{S}_{n}} E(X, Y) \text {; }
$$

and the second player can insure that the expectation of the first will be at most

$$
\min _{Y \in S_{n}} \max _{x \in S_{m}} E(X, Y) .
$$

At this point, however, the situation diverges radically from the case of pure strategies, for now it can be shown that, for every payoff matrix $M$, we have

$$
\max _{X \in \mathcal{S}_{m}} \min _{Y \in \mathcal{S}_{n}} E(X, Y)=\min _{Y \in \mathcal{S}_{n}} \max _{X \in \mathcal{S}_{m}} E(X, Y) .
$$

Thus if we permit the players to use mixed strategies, then every finite zero-sum, two-person game has optimum strategies for the two players. This theorem, which was first proved by von Neumann, ${ }^{7}$ is sometimes called the "minimax theorem"; it is the fundamental theorem of finite games.

7 The earliest proof of this theorem is to be found in von Neumann [19]; this proof makes use of the Brouwer fixed-point theorem. An elementary algebraic proof was given later in Ville [24]. In von Neumann and Morgenstern [20, pp. 153-158], will be found a simple proof depending on the theory of convex sets. Other proofs are to be found in Loomis [14] and Brown and von Neumann [6]. 
Thus if we let $\mathscr{D}_{1}$ and $\mathscr{D}_{2}$ be the sets of optimum mixed strategies for $P_{1}$ and $P_{2}$ respectively, we see that $\mathcal{D}_{1}$ and $\mathcal{D}_{2}$ are not empty. Since $\mathcal{D}_{1} \subseteq S_{m}$ and $\mathscr{D}_{2} \subseteq S_{n}$, it is clear that $\mathscr{D}_{1}$ and $\mathscr{D}_{2}$ are respectively subsets of $(m-1)$-dimensional and $(n-1)$-dimensional Euclidean space. It is easily verified that $\mathscr{D}_{1}$ and $\mathscr{D}_{2}$ are closed and convex; and a proof that $\mathscr{D}_{1}$ and $\mathscr{D}_{2}$ are polyhedral has been given by Shapley and Snow (in [22]), who have also established a constructive method for finding the vertices of the polyhedra; from the convexity this of course enables one to find all the members of $\mathscr{D}_{1}$ and $\mathscr{D}_{2}$. It should be remarked, however, that the method of Shapley and Snow becomes laborious in the case of games with matrices of large order, since the method involves inverting a substantial proportion of the submatrices of the matrix of the game.

A more rapid (iterative) procedure for computing a pair of optimum strategies for a given game has been developed by Brown (see [5]), and shown to be convergent by Julia Robinson. ${ }^{8}$

Mathematicians have also interested themselves in the problem of what sets $\mathcal{D}_{1}$ and $\mathcal{D}_{2}$ can be regarded as the sets of optimum strategies for the two players of some zero-sum, two-person game. This problem has an elegant solution, which has been found by Bohnenblust, Karlin, and Shapley (in [3]), and independently by Gale and Sherman (in [8]).

Although the theory of finite zero-sum two-person games might thus seem to be already in a satisfactory form, it should be noticed that neither of the above methods of finding optimum mixed strategies is practicable when the number of pure strategies is more than, say, 100. On the other hand, when we normalize almost any game given in extensive form, the number of pure strategies is found to be quite large. Thus, even for the very simple game of ticktacktoe, there are more than $10^{10,000}$ pure strategies for each player; and clearly the number of pure strategies increases at a tremendous rate with the number of moves in the game. Two partial solutions have been given for the difficulties that arise from this circumstance.

The first partial solution rests on the fact that certain information in a game may be in a sense superfluous. Thus suppose, for example, that the first two moves of a certain game are both made by $P_{1}$, and that $P_{1}$, when he makes his second move, is informed what he did on his first move; then we see that this information is actually of no help to $P_{1}$, since his knowing what strategy he is playing insures that he knows what he did on his first move; thus we can obtain an equivalent game by decreasing the information given $P_{1}$ in his second

${ }^{8}$ See Robinson [21]. 
move, which will decrease the number of information sets for $P_{1}$, and hence the number of strategies available to him. A simple method of deciding what information sets can safely be coalesced has been given by Krentel, McKinsey, and Quine (in [11]); this method, however, applies only to a certain subclass of the totality of finite games. An interesting condition applying to all finite games has recently been found by Norman Dalkey; ${ }^{9}$ in this general case, however, there may be more than one game which is equivalent to a given game, and which is "completely deflated" (i.e., in which no more information sets can be coalesced without losing equivalence); and the problem remains to pick out, from among the completely deflated games equivalent to a given game, one which will make the number of pure strategies as small as possible.

The second partial solution, which goes in a different direction altogether, was first made use of by Morgenstern and von Neumann (see [20, pp. 186-218]) in a discussion of a certain form of poker, and was later treated in a general way by Kuhn in [12]. In order to explain this method, it is necessary to introduce two new notions about games. By a game with perfect recall we mean a game in which each player, at each of his moves, remembers everything he did and knew at his previous moves; thus every two-person game which can be played by just two people (rather than by teams) is a game with perfect recall; rummy, for instance, is a game with perfect recall, but bridge is not-since in bridge each player is a pair of people, neither of whom is informed what cards the other holds. (I have given here only an intuitive explanation of this notion; it can easily be made mathematically precise, however, by expressing a condition on the information partition for the given game.) By a behavior strategy for a player of a game is meant a set of probability distributions, one for each of the player's information sets; and such that, if $\alpha$ is an information set which presents $r$ alternatives, then the probability distribution corresponding to $\alpha$ is a member of $S_{r}$. It is clear that, instead of using mixed strategies in playing a given game, one can also use behavior strategies; it is intuitively evident, moreover, that one can always do at least as well with a mixed strategy as with a behavior strategy. Now Kuhn ${ }^{10}$ has shown, conversely, that, in the case of a game with perfect recall, one can always do as well with a

${ }^{9}$ This result is to appear in the forthcoming second volume of Contributions to the theory of games (Annals of Mathematics Studies, No. 28), which will be published by the Princeton University Press.

${ }^{10}$ See Kuhn [12]. Actually Kuhn established a considerably stronger result than the one stated above. 
behavior strategy as with a mixed strategy. This fact greatly decreases the difficulty of calculating optimum ways of playing games with perfect recall, for in general the number of parameters to be determined in calculating optimum behavior strategies is much smaller than the number of parameters to be determined in calculating optimum mixed strategies.

It should be remarked that the above two methods cannot very well be used in combination. For the first method, which coalesces some of the information sets of a player, leads from games with perfect recall to games without perfect recall. Thus the question arises concerning when it is better to use one method, and when the other. In general, it appears that some more attention could profitably be devoted to the problem of how to solve games given in extensive form.

I turn now to games which are not finite: for example, to games in which choices are to be made from an infinite, instead of from a finite, number of alternatives. A great part of the research in this direction has been devoted to games which are already in normalized form. Thus suppose that $P_{1}$ chooses a real number $x$ from the closed unit interval $(0 \leqq x \leqq 1)$, that $P_{2}$, without being informed what choice $P_{1}$ has made, then chooses a number $y$ from the closed unit interval, and that $P_{2}$ then pays $P_{1}$ an amount $M(x, y)$, where $M$ is a function of two real variables defined over the closed unit square. Such games are called continuous games. By a mixed strategy for such a game is meant a cumulative distribution function over the unit interval. If $P_{1}$ chooses $x$ by means of a cumulative distribution function $f$, and $P_{2}$ chooses $y$ by means of a cumulative distribution function $g$, then the expectation $E(f, g)$ of $P_{1}$ is given by the formula

$$
E(f, g)=\int_{0}^{1} \int_{0}^{1} M(x, y) d f(x) d g(y) .
$$

It has been shown by Ville (in [24]) that if $M$ is continuous, then

$$
\max _{f} \min _{0} E(f, g)=\min _{0} \max _{f} E(f, g),
$$

so that a continuous game with a continuous payoff function has optimum mixed strategies for the two players. This result has been strengthened and generalized by Wald (in [25]), and by Karlin (in [10]).

As might be expected, mathematicians have devoted considerable attention to the problem of finding optimum mixed strategies for particular continuous games. Interesting results of this sort have 
been obtained in two rather wide special cases: (1) the case where $M(x, y)$ is convex in $y$ for each $x$ (and the analogous case where $M(x, y)$ is concave in $x$ for each $y)$; and (2) the case where $M(x, y)$ is a polynomial. In the first case, it has been shown by Bohnenblust, Karlin, and Shapley (in [4]) that if $M(x, y)$ is strictly convex in $y$ for each $x$, and is differentiable over the closed unit square, then there is a unique optimum strategy for $P_{2}$, which assigns probability 1 to one point, and hence probability 0 to all other points (thus what is called a step-function with one step) and there is a step-function with two steps which is an optimum strategy for $P_{1}$. In the second case also, which has been treated by Dresher, Karlin, and Shapley (in [7]), it has been shown that each player always has an optimum strategy which is a step-function (the number of steps depending on the degree of $M$ ); and constructive methods have been developed for finding the appropriate step-functions. The methods of Dresher, Karlin, and Shapley are also applicable to many games with payoff functions of the type called polynomial-like: i.e., which can be represented in the form

$$
M(x, y)=\sum_{j=1}^{n} \sum_{i=1}^{m} a_{i} r_{i}(x) s_{j}(y)
$$

where $r_{1}, \cdots, r_{m}$ and $s_{1}, \cdots, s_{n}$ are continuous.

There are also many interesting rather special results known in this connection. Gross ${ }^{11}$ has given an example of a continuous game with a rational payoff function which has no step-function as an optimum strategy. Blackwell and Girshick have given an example ${ }^{12}$ of a continuous game where every pure strategy is employed in the unique optimum strategy. Bellman and Blackwell (in [2]) have given an interesting analysis of the role of bluffing in two-person poker.

It goes without saying that there remain a great variety of unsolved problems in this domain. Formulations of some of these will be found in Kuhn and Tucker's introduction to a recent collection of papers on games (see [13]).

A problem which appears to be particularly important, but unfortunately very difficult, is that of dealing in some way with games which have more than one move, and where the choices are made from infinite sets. Thus suppose, for instance, that a game has four moves: in the first move $P_{1}$ chooses a number $x_{1}$ from the unit interval; in the second move, $P_{2}$, knowing $x_{1}$, chooses a number $y_{1}$ from the unit

11 See Gross [9]. This proof makes use of the fact, established in Tarski [23], that only algebraic numbers are definable in elementary algebra.

${ }^{12}$ In a private communication. 
interval; in the third move, $P_{1}$, knowing $y_{1}$, but having forgotten $x_{1}$, chooses a number $x_{2}$ from the unit interval; and in the fourth move $P_{2}$, knowing $y_{1}$ and $x_{2}$, but not $x_{1}$, chooses a number $y_{2}$ from the unit interval. (The payoff is then some function of the four variables $x_{1}, x_{2}, y_{1}, y_{2}$. ) A pure strategy for $P_{1}$ is now an ordered couple $\langle a, f\rangle$, where $a$ is a real number and $f$ is a function of one variable (it depends on $\left.y_{1}\right)$; and a pure strategy for $P_{2}$ is an ordered couple $\langle g, h\rangle$, where $g$ is a function of one variable (it depends on $x_{1}$ ) and $h$ is a function of two variables (it depends on $y_{1}$ and $x_{2}$ ). Thus in this game a mixed strategy is a distribution function over function space. It is not clear even over which subsets of this function space the probability distributions are to be regarded as being defined; and certainly we do not know conditions under which optimum strategies for such a game exist, or how to find them when they do.

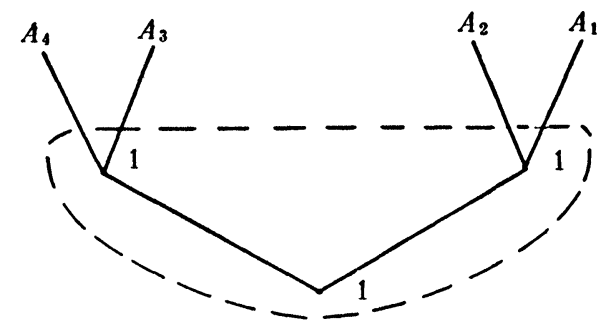

FIG II

Another important problem, to which attention has been called by Helmer, ${ }^{12}$ is the problem of finding some rational way of dealing with conflict situations which are not technically two-person zerosum games, though they closely resemble such games. I shall begin with a detailed examination of a situation which violates Condition A above. Just in order to have a term, let us agree to mean by a "pseudo-game" something that is like a game, except that Condition $A$ is not satisfied. A very simple pseudo-game is one whose graphical representation is given in Figure II. Here we can suppose, if we please, that there are two players, $P_{1}$ and $P_{2}$; but all the moves are made by $P_{1}$. On the first move, $P_{1}$ is to choose from two alternatives, and on the second move he is again to choose from two alternatives. The dotted line enclosing the three vertices indicates the sole information set of the game; thus $P_{1}$, when he moves, does not know whether he is moving for the first or the second time. Since plays intersect the information set in two points, Condition $\mathrm{A}$ is not satisfied, and hence this is only a pseudo-game.

It is apparent that, in order to realize this situation in practice, it 
would be necessary to make $P_{1}$ a team consisting of two human beings. For if $P_{1}$ consisted of a single human being, then, on the second move, $P_{1}$ would remember that he had already moved, so that his information would be better than that indicated by the diagram.

Hence, suppose that there are two men, $P_{1}^{(1)}$ and $P_{1}^{(2)}$, who are together to constitute $P_{1}$, and suppose they are put into separate rooms and not allowed to communicate with each other during the play. After the beginning of the play, the umpire is to go into one room, and ask the man there to choose one of the alternatives I and II, and then into the other room, and ask the other man to choose one of the alternatives I and II. $P_{2}$ will then pay $P_{1}$ an amount $M(x, y)$, where $x$ is the alternative chosen by the man in the first room, and $y$ is the alternative chosen by the man in the second room.

If we were to define a strategy for a pseudo-game in the same way we defined it for a game, then there would be only two strategies for $P_{1}$ in this game: one strategy would make him always pick I, and the other would make him always pick II. The first strategy would make him always end up at $A_{1}$, and the second would make him always end up at $A_{4}$. Any mixed strategy, consequently, would make him end up sometimes at $A_{1}$ and sometimes at $A_{4}$-but never at $A_{2}$ or $A_{3}$. Since it can happen, however, that the payoffs to $P_{1}$ at $A_{2}$ and $A_{3}$ are both higher than at $A_{1}$ or $A_{4}$, it is seen that in some cases $P_{1}$ can do better than by playing any mixed strategy: for example $P_{1}^{(1)}$ and $P_{1}^{(2)}$ could agree that $P_{1}^{(1)}$ is to choose $\mathrm{I}$, and $P_{1}^{(2)}$ is to choose II - in which case the play would terminate either at $A_{\mathbf{2}}$ or $A_{3}$.

Thus it might be thought that for this game we should distinguish four strategies:

1. $P_{1}^{(1)}$ and $P_{1}^{(2)}$ both pick I;

2. $P_{1}^{(1)}$ and $P_{1}^{(2)}$ both pick II;

3. $P_{1}^{(1)}$ picks I, and $P_{1}^{(2)}$ picks II;

4. $P_{1}^{(1)}$ picks II, and $P_{1}^{(2)}$ picks I.

But now the difficulty arises that, if $P_{1}$ plays strategy 3 or 4 , then it is impossible to tell whether the play will terminate at $A_{2}$ or at $A_{3}$; for this will depend on whether the umpire goes first into the room occupied by $P_{1}^{(1)}$ or first into the room occupied by $P_{1}^{(2)}$. Since the payoffs at $A_{2}$ and $A_{3}$ are not necessarily equal, however, and since we do not know even the probability that the umpire will go first into the room occupied by $P_{1}^{(1)}$, we cannot in general calculate the expectation of $P_{1}$ if strategy 3 , or strategy 4 , is used. Thus our theory of games cannot be carried over in any obvious way to pseudogames.

It is in order to avoid difficulties of this sort that we require 
that games satisfy Condition $\mathrm{A}$. By imposing this condition we reduce the theory of games to something manageable; but the difficulties have not been legislated out of existence: they continue to exist, even if no longer countenanced as part of the theory of games. For conflict situations describable only as pseudo-games do arise in practice, and we sometimes have to cope with them-regardless of what we choose to call them. Suppose, for example, that two policemen, $A$ and $B$, are trying to shoot an escaped insane criminal, $C$. Here it is natural to regard $A$ and $B$ as one "player," and $C$ as the other "player." $A$ and $B$ may find it advantageous to separate; and after they have done so, $A$, when he sees $C$ and shoots at him, may very well not know whether $B$ has previously shot. It does not seem unreasonable to inquire what sort of actions $A$ and $B$ should take, even though this is not technically a game. This class of problems seems to be equivalent to the problem of defining optimum strategies for games in normalized form, where the values of the elements of the payoff matrix are not known exactly, but are merely required to satisfy certain inequalities.

It can also happen that, due for instance to difficulties of communication, it is not possible for the various members of a team to agree upon a strategy before the beginning of a play-in which case again, the theory of games in normalized form is no longer applicable. Situations of this sort are especially apt to occur in connection with military affairs.

In concluding this survey, I turn to a consideration of the problems which arise in connection with what are called "general" games: i.e., games which are not necessarily zero-sum, and which may have more than two players. As has been mentioned earlier, such games are particularly important so far as regards practical applications, but they present great conceptual difficulties; in particular, game theorists have not been able even to come to any general agreement as to what should be meant by a solution of such a game.

I shall discuss briefly the two most ambitious attempts ${ }^{13}$ that have been made to deal with this subject: that of von Neumann, and that of Nash. I shall not try to give anything like a complete and adequate account of the theories in question, however, but shall merely say enough to give an intuitive idea of them.

von Neumann's treatment $t^{14}$ of general games is based on the notion

${ }^{13}$ More recently a very interesting partial theory of zero-sum $n$-person games has been developed by L. S. Shapley. This theory was outlined by Shapley at the September 4, 1951 meeting of the Econometric Society at Minneapolis. A brief account will be found in Econometrica vol. 20 (1952) p. 91.

14 Roughly the last two-thirds of von Neumann and Morgenstern [20] is devoted to an exposition of this theory of general games. 
of a characteristic function for such a game, which is introduced as follows. If $N$ is the set of all players of a game, and if $S$ is any subset of $N$, then it can happen that the players form themselves into two "coalitions," $S$ and $N-S$, which cooperate among themselves and oppose each other. If this happens, then the game becomes essentially a two-person game, and we can define a function $v$ by agreeing that if $S$ is any subset of $N$, then $v(S)$ is the total amount the members of $S$ could expect to obtain, if they all cooperated together, and all the other players formed themselves into an opposing coalition, and tried to hurt the members of $S$ as much as possible.

By a solution of a general $n$-person game, von Neumann means a certain set of ordered $n$-tuples of real numbers. I am not going to repeat here the exact conditions which must be satisfied by such a class of $n$-tuples, except to remark that the conditions are expressed solely in terms of the characteristic function; so that two games whose characteristic functions coincide will have the same solutions. The intuitive meaning of a solution is roughly the following: it represents the set of all possible ways, for a given organization of society, in which money can be distributed among the players at the end of a play.

Now consider a two-person non-zero-sum game in normalized form, where $P_{1}$ has just one strategy (so the outcome of a play cannot be affected by $\left.P_{1}\right)$, and $P_{2}$ has two strategies; and suppose that, in case $P_{2}$ uses his first strategy, then the payoff to $P_{1}$ is 10 , and to $P_{2}$ is 0 ; and in case $P_{2}$ uses his second strategy, the payoff to $P_{1}$ is 0 and to $P_{2}$ is $-1,000$. It is convenient to represent such games by a pair of payoff matrices:

$$
\begin{array}{cc}
\text { Payoff to } P_{1} & \text { Payoff to } P_{2} \\
(100) & (0-1,000) .
\end{array}
$$

Thus we again think of $P_{1}$ as being able to choose a row (but there in only one row in this case, so he has no real choice), and of $P_{2}$ as being able to choose a column.

The characteristic function of this game is a function defined over the four subsets of the set of two players. Since neither $P_{1}$ nor $P_{2}$ can, through his own endeavors, make sure of getting more than 0 , we see that

$$
v\left(\left\{P_{1}\right\}\right)=v\left(\left\{P_{2}\right\}\right)=0 .
$$

Moreover, $P_{1}$ and $P_{2}$ can together behave in such a way that the sum of the payments to them will be 10 (in order to achieve this end, it is only necessary that $P_{2}$ choose the first column); hence 


$$
v\left(\left\{P_{1}, P_{2}\right\}\right)=10 .
$$

Finally, as in any game, if $\Lambda$ is the empty set, we have

$$
v(\Lambda)=0 .
$$

Equations (3), (4), and (5) completely specify the characteristic function.

For this game it turns out that there is just one solution in the sense of von Neumann, and this solution is the set of all couples $\left\langle x_{1}, x_{2}\right\rangle$ of non-negative real numbers such that $x_{1}+x_{2}=10$. This may be interpreted in the following way: before playing this game the two players will (or perhaps merely "should") get together and agree that $P_{2}$ will choose the first column, so that $P_{1}$ will be paid 10 and $P_{2}$ will be paid 0 ; they will decide, moreover, how this 10 is to be divided between them into $x_{1}$ and $x_{2}$; this division can be arbitrary, subject only to the restriction that $x_{1}$ and $x_{2}$ are both to be nonnegative and are to have a sum of 10.

Two objections have been urged against von Neumann's notion of a solution. In the first place, it is felt by some people that knowing the solution in this sense of the word would not be much help in playing the game. The solution leaves a wide range of indeterminancy, and tells us nothing about the way in which the agreement between $P_{1}$ and $P_{2}$ is finally to be reached.

Secondly, it seems doubtful that the characteristic function adequately represents all the complications of the game. Thus, for example, one has the intuitive feeling that, in the game described above, $P_{1}$ is in a better bargaining position than is $P_{2}$, and most people feel they would rather play the part of $P_{1}$ than that of $P_{2}$ : for $P_{1}$ will automatically be paid 10, unless $P_{2}$ is willing to injure himself very greatly by choosing the second column, and hence himself losing 1,000 ; therefore it hardly seems likely that $P_{2}$ will be able to persuade $P_{1}$ to pay him part of the 10 , in order to insure that $P_{2}$ will play the first column. On the other hand, the characteristic function of this game, from (3), is symmetric in $P_{1}$ and $P_{2}$; so that no asymmetry can appear in any notion of a solution, so long as the solution is defined solely in terms of the characteristic function.

An attempt to avoid these difficulties, and to devise an altogether new way of dealing with general games, has been made by Nash (in [18]). Nash distinguishes first between what he calls "noncooperative" and "cooperative" games. A noncooperative game is one in which no communication is allowed between the players, and in which, in particular, they are not allowed to make agreements about 
side-payments; a cooperative game is one in which communication is allowed.

For the noncooperative game, Nash introduces the notion of an equilibrium point, which is a generalization of the notion of a saddlepoint for a two-person zero-sum game. An equilibrium point for an $n$-person game is an ordered $n$-tuple $\left\langle x_{1}, \cdots, x_{n}\right\rangle$ of strategies such that, for $i=1, \cdots, n$, if every player $P_{j}$, for $j \neq i$, chooses $x_{j}$, then $P_{i}$ cannot do better than to choose $x_{i}$. Nash shows that every finite game has an equilibrium point (in mixed strategies). He also points out that social groups who repeatedly play a certain game often fall into the habit of playing an equilibrium point; in such cases, of course, a newcomer to the group (unless he is able to persuade others to deviate) cannot do better than to stay with the equilibrium point also, and in general he will lose if he deviates from it.

Nash calls an $n$-person game solvable in case the set, $S$, of all its equilibrium points satisfies the following condition: if $1 \leqq i \leqq n$, and if $\left\langle x_{1}, \cdots, x_{n}\right\rangle \in S$ and $\left\langle y_{1}, \cdots, y_{n}\right\rangle \in S$, then $\left\langle x_{1}, \cdots, x_{i-1}\right.$, $\left.y_{i}, x_{i+1}, \cdots, x_{n}\right\rangle \in S$. If a game is solvable in this sense, the set of its equilibrium points is called its solution. He deals with the cooperative games, in turn, by reducing them to noncooperative games in the following way: the negotiations of the cooperative game are included as formal moves in a noncooperative game (these moves consist of such procedures as, for example, one player's making an offer of a side-payment to another).

It must be remarked that Nash's theory-though it represents a considerable advance - has some grave deficiencies and certainly cannot be regarded as a definitive solution of the conceptual problems of this domain. In the first place, so far as regards the noncooperative games, it is unfortunately not the case that every game is solvable in the sense of Nash. Thus consider the two-person game whose matrices are as follows:

$$
\begin{array}{rr}
\text { Payoff to } P_{1} & \text { Payoff to } P_{2} \\
\left(\begin{array}{rr}
10 & -30 \\
8 & -18
\end{array}\right) & \left(\begin{array}{rr}
-20 & -30 \\
6 & 12
\end{array}\right) .
\end{array}
$$

Here there is an equilibrium point in the upper left-hand corner, and one in the lower right-hand corner; but the other two points in the matrices are not equilibrium points, and hence the game is not solvable. $P_{1}$ would of course prefer the equilibrium point in the upper left-hand corner, and $P_{2}$ would prefer the equilibrium point in the lower right-hand corner. The theory of Nash seems to throw little 
light on the question how to play a game having such a pair of payoff matrices.

In the second place, even if the theory of noncooperative games were in a completely satisfactory state, there appear to be difficulties in connection with the reduction of cooperative games to noncooperative games. It is extremely difficult in practice to introduce into the noncooperative games the moves corresponding to negotiations, in a way which will reflect all the infinite variety permissible in the cooperative game, and this without giving one player an artificial advantage (due to his having the first chance of making an offer, let us say).

Thus it seems that, despite the great ingenuity that has been shown in the various attacks on the problem of general games, we have not yet arrived at a satisfactory definition of a solution of such games. It is rather likely, as has been suggested by Bellman, ${ }^{12}$ that it will be found necessary to distinguish many types of games, and define "solution" differently for different types; the theory of Nash of course represents a step in this direction. This whole aspect of the theory of games presents a challenging problem to the mathematician, and in my opinion an extremely important one-since the application of game theory to a very wide class of practical situations must wait for such a definition.

\section{BIBLIOGRAPHY}

1. K. J. Arrow, D. Blackwell, and M. A. Girshick, Bayes and minimax solutions of sequential decision problems, Econometrica vol. 17 (1949) pp. 213-244.

2. R. Bellman and D. Blackwell, Some two-person games involving bluffing, Proc. Nat. Acad. Sci. U.S.A. vol. 35 (1949) pp. 600-605.

3. H. F. Bohnenblust, S. Karlin, and L. S. Shapley, "Solutions of discrete twoperson games," Contributions to the theory of games (ed. by H. W. Kuhn and A. W. Tucker), Princeton, Princeton University Press, 1950.

4. - "Games with continuous, convex pay-off," Contributions to the theory of games (ed. by H. W. Kuhn and A. W. Tucker), Princeton, Princeton University Press, 1950.

5. G. W. Brown, Iterative solutions of games by fictitious play, Activity Analysis of Production and Allocation, Cowles Commission Monograph No. 13, New York, Wiley, 1951.

6. G. W. Brown and J. von Neumann, "Solutions of games by differential equations," Contributions to the theory of games (ed. by H. W. Kuhn and A. W. Tucker), Princeton, Princeton University Press, 1950.

7. M. Dresher, S. Karlin, and L. S. Shapley, "Polynomial games," Contributions to the theory of games (ed. by H. W. Kuhn and A. W. Tucker), Princeton, Princeton University Press, 1950.

8. D. Gale and S. Sherman, "Solutions of finite two-person games," Contributions to the theory of games (ed. H. W. Kuhn and A. W. Tucker), Princeton, Princeton University Press, 1950. 
9. O. Gross, "On certain games with transcendental value," Contributions to the theory of games. II, Annals of Mathematics Studies, No. 28, in preparation.

10. S. Karlin, "Operator treatment of minimax principle," Contributions to the theory of games (ed. by H. W. Kuhn and A. W. Tucker), Princeton, Princeton University Press, 1950.

11. W. D. Krentel, J. C. C. McKinsey, and W. V. Quine, A simplification of games in extensive form, Duke Math. J. vol. 18 (1951) pp. 885-900.

12. H. W. Kuhn, Extensive games, Proc. Nat. Acad. Sci. U.S.A. vol. 36 (1950) pp. 570-576.

13. H. W. Kuhn and A. W. Tucker, "Preface" to Contributions to the theory of games, Princeton, Princeton University Press, 1950.

14. L. H. Loomis, On a theorem of von Neumann, Proc. Nat. Acad. Sci. U.S.A. vol. 32 (1946) pp. 213-215.

15. J. McDonald, The theory of strategy, Fortune vol. 38 (1949) pp. 100-110.

16. - Strategy in poker, business, and war, New York, Norton, 1950.

17. P. M. Morse, Mathematical problems in operations research, Bull. Amer. Math. Soc. vol. 54 (1948) pp. 602-621.

18. J. F. Nash, Non-cooperative games, Ann. of Math. vol. 54 (1951) pp. 286-295.

19. J. von Neumann, Zur Theorie der Gesellshaftsspiele, Math. Ann. vol. 100 (1928) pp. 295-320.

20. J. von Neumann and O. Morgenstern, Theory of games and economic behavior, Princeton, Princeton University Press, 1947.

21. J. Robinson, An iterative method of solving a game, Ann. of Math. vol. 54 (1951) pp. 296-301.

22. L. S. Shapley and R. N. Snow, "Basic solutions of discrete games," Contributions to the theory of games (ed. by H. W. Kuhn and A. W. Tucker), Princeton, Princeton University Press, 1950.

23. A. Tarski, $A$ decision method for elementary algebra and geometry, Berkeley, University of California Press, 1951.

24. J. Ville, "Sur la théorie générale des jeux où intervient l'habilité des jeueurs," Traite du Calcul des Probabilités et ses Applications, by E. Borel and collaborators, Paris, 1938, vol. 2, pp. 105-113.

25. A. Wald, Foundation of a general theory of sequential decision functions, Econometrica vol. 15 (1947) pp. 279-313.

26. - Statistical decision functions, New York, Wiley, 1950.

\section{STANFORD UNIVERSITY}

